\title{
Thermo-responsive hydrogel as an anti-VEGF drug delivery system to inhibit retinal angiogenesis in Rex rabbits
}

\author{
Chao-Chien $\mathrm{Hu}^{\mathrm{a}, \mathrm{b}}$, Yin-Chen Chiu ${ }^{\mathrm{c}}$, Jen-Ray Chaw ${ }^{\mathrm{c}}$, Chin-Fu Chen ${ }^{\mathrm{d}}$ and Hsia-Wei Liu ${ }^{\mathrm{e}, *}$ \\ ${ }^{a}$ Department of Ophthalmology, Shin Kong Wu Ho-Su Memorial Hospital, Taipei, Taiwan \\ ${ }^{\mathrm{b}}$ School of Medicine, Fu Jen Catholic University, New Taipei City, Taiwan \\ ${ }^{\mathrm{c}}$ Graduate Institute of Applied Science and Engineering, Fu Jen Catholic University, New Taipei City, \\ Taiwan \\ ${ }^{\mathrm{d}}$ Biomedical Technology and Device Research Laboratories, Industrial Technology Research Institute, \\ Taiwan \\ ${ }^{\mathrm{e}}$ Department of Life Science, Fu Jen Catholic University, New Taipei City, Taiwan
}

\begin{abstract}
.
BACKGROUND: Wet age-related macular degeneration (Wet AMD) has been treated clinically by intravitreal injection of bevacizumab, which is a kind of the anti-VEGF antibody drug. Nevertheless, because of the short half-life and frequent injections, the use of this treatment is limited.

OBJECTIVE: To confirm whether mPEG-PLGA-BOX can be considered as a VEGF drug delivery system to inhibit retinal angiogenesis.

METHODS: A thermo-responsive hydrogel of methoxy-poly (ethylene glycol)-block-poly (lactic-co-glycolic acid) (mPEGPLGA-BOX) was synthesized. The thermo-responsive hydrogel mPEG-PLGA-BOX was able to have sol-gel phase transition upon stimulation by the body temperature with improved biocompatibility and biodegradation. The bevacizumab released from mPEG-PLGA-BOX inhibited RF/6A cells according to a JC-1 assay, which indicated that the released bevacizumab was active to be able to suppress the growth of new blood vessels. In an animal study, retinal laser photocoagulation was performed to induce angiogenesis in the eyes of Rex rabbits using an 810-mm laser.

RESULTS: The retina was penetrated when the laser power was more than $500 \mathrm{~mW}$ and the exposure time was more than $500 \mathrm{~ms}$. New blood vessels were created at the 28th day after retinal laser photocoagulation. At this time, intravitreal 0.05-mL injections of mPEG-PLGA-BOX (bevacizumab) solution were administered. The bevacizumab released from mPEG-PLGABOX (bevacizumab) solution suppressed the angiogenesis. In an in vivo study, the histomorphology of the rabbit retina also indicated that mPEG-PLGA-BOX after intravitreal injection is not toxic to the rabbit retina.

CONCLUSIONS: Bevacizumab released from mPEG-PLGA-BOX (bevacizumab) solution suppressed angiogenesis, and mPEG-PLGA-BOX can be considered as a novel thermo-responsive hydrogel with potential as a gelling carrier for extended bevacizumab drug release to treat intraocular neovascular diseases.
\end{abstract}

Keywords: Bevacizumab, thermo-responsive hydrogel, intraocular neovascular diseases, angiogenesis

${ }^{*}$ Corresponding author: Hsia-Wei Liu, Department of Life Science, Fu Jen Catholic University, New Taipei City, Taiwan. Tel.: +886 910688968; Fax: +886 229053740; E-mail: 079336@ mail.fju.edu.tw.

0928-7329/19/\$35.00 (c) 2019 - IOS Press and the authors. All rights reserved

This article is published online with Open Access and distributed under the terms of the Creative Commons Attribution NonCommercial License (CC BY-NC 4.0). 


\section{Introduction}

The exudative form of AMD (wet AMD) is caused by an increase of vascular endothelium growth factor (VEGF). Among elderly people in developed countries, irreversible vision loss (even blindness) is primarily caused by AMD [1,2]. In the past decade, intravitreal injections of anti-VEGF agents have been an effective main clinical treatment that is widely used for neovascular AMD. Such agents include bevacizumab and ranibizumab [3-5].

Bevacizumab must be stored at temperatures of 2 to $8^{\circ} \mathrm{C}$ and kept away from light [6]. The activity of anti-VEGF in bevacizumab may degrade gradually due to the body temperature after intravitreal injections and even when stored at $4^{\circ} \mathrm{C}$ [7]. The anti VEGF method to treat AMD is limited due to the degradation of anti-VEGF activity at body temperature, which may shorten the half-life of bevacizumab. Because of the very short half-life (4.25 days) [8], the major concern of anti-VEGF drugs for treating neovascular AMD is frequent intravitreal injections [9]. The development of an effective drug carrier to make bevacizumab delivery long lasting and less invasive could enhance a safer and more effective clinical application in the current therapy for wet AMD and other neovascular retinal disorders. Swellable polymeric nanomaterials such as nanogels and liposomes have been widely investigated as carriers for drug delivery systems and are widely applied in medicine [10-12].

Biodegradable thermo-sensitive polymers that have sol-gel-sol transition characteristics with increasing temperature have been widely studied for drug delivery [13]. These polymers have several advantages compared to other biodegradable polymers [14,15], such as high drug-encapsulation efficiency, longer period of drug release, and easy preparation. Appropriate sol-gel transition behavior is requested in an ideal biodegradable thermo-sensitive hydrogel for drug delivery, which means an ideal biodegradable thermo-sensitive must be in a solution phase at room temperature and in a gel phase at body temperature.

Since 2012, an amphiphilic and biodegradable thermo-sensitive PEOz-PCL-PEOz triblock copolymer has been applied to carrier and release bevacizumab in the intravitreal injections [16]. In 2014, another amphiphilic thermo-sensitive nanogel was used as a drug delivery system to carry bevacizumab for intravitreal injections. In our previous research, we have confirmed that bevacizumab is captured in the thermo-sensitive hydrogel mPEG-PLGA-BOX when the operation temperature is above the lower critical solution temperature (LCST). We also conformed bevacizumab is released after the intravitreal injection [17]. mPEG-PLGA-BOX was proven to be a suitable thermo-sensitive nanogel system. The bioactivity of bevacizumab was also evaluated upon release using the human umbilical vein endothelial cells and the rhesus choroids-retina endothelial cell line. In an in vitro study in 2015, the release rate of bevacizumab in mPEG-PLGA-BOX was also confirmed [18].

Poly (-ethylene glycol) diacrylate hydrogels (PEGDA) can be designed to provide exhibit patterned strain and anisotropic structures under mechanical loading. PEGDA hydrogels with photolithographic patterning techniques and varying polymer chain length were used to render substrates with tunable mechanical properties and spatially patterned. PEGDA can also be applied to study the cell response to substrate rigidity in two and even three dimensions [19]. The purpose of this study is to find out the effect of angiogenesis inhibition for bevacizumab released from mPEG-PLGA-BOX. This was done through an in vitro study in which rhesus choroid-retina endothelial cells and human fibroblasts were cultured in PEGDA. The apoptosis of the rhesus choroids-retina endothelial cells was examined using a JC-1 assay. An in vivo animal study was also done to investigate whether the released bevacizumab still suppressed angiogenesis. 

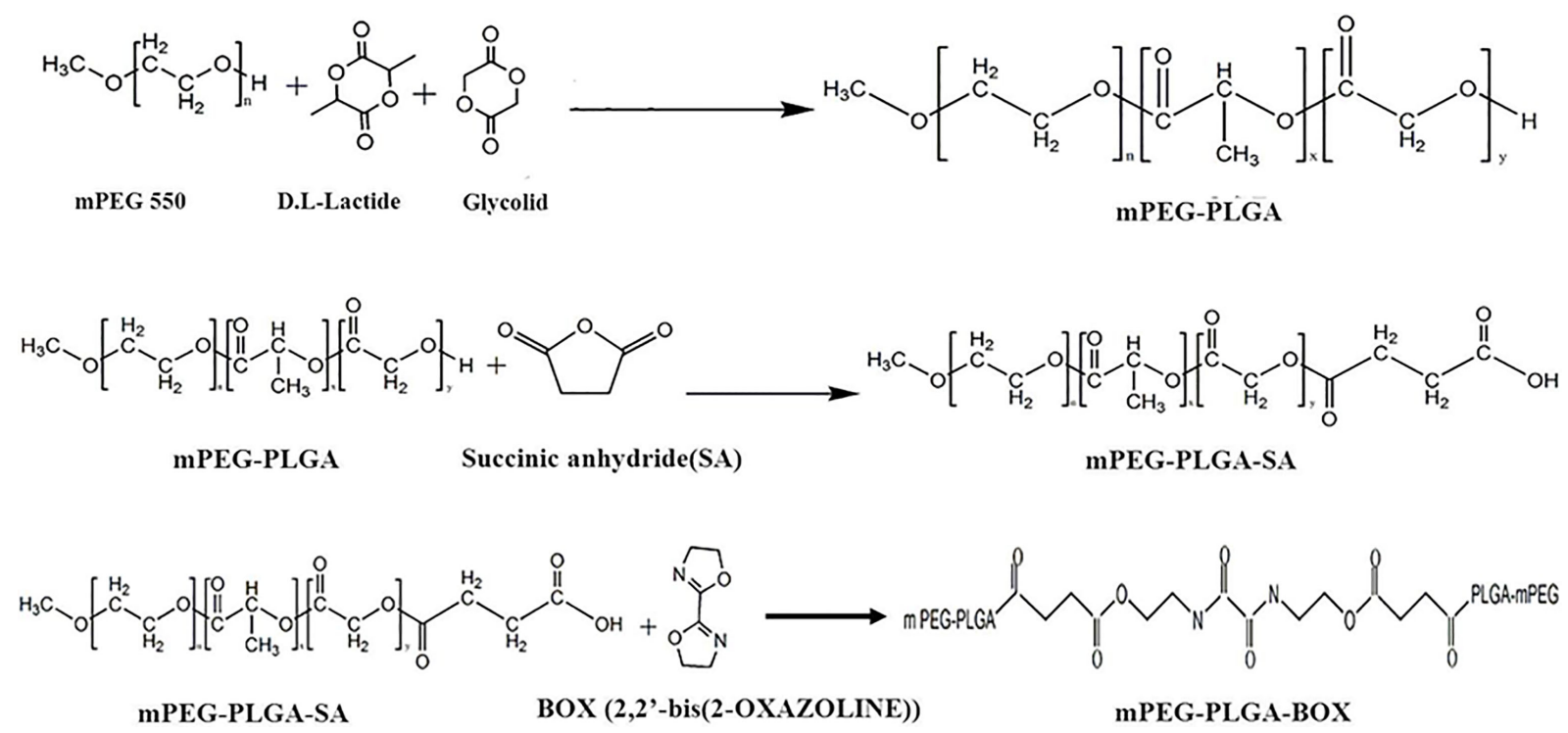

Scheme 1. Synthesis scheme of mPEG-PLGA-BOX.

\section{Materials and methods}

\subsection{MPEG-PLGA-BOX diblock copolymer preparation}

A thermo-responsive hydrogel of methoxy-poly (ethylene glycol)-block-poly (lactic-co-glycolic acid) (mPEG-PLGA-BOX) was synthesized through three steps (Scheme 1). For the first step, mPEG solution was mixed with glycolide (GA), D, L-lactide (LA), and $0.5 \mathrm{~mL}$ of a $0.1 \mathrm{M}$ solution of tin (II) 2ethylhexanoate in dried toluene under nitrogen at room temperature. The reaction of copolymer $\mathrm{mPEG}-$ PLGA proceeded at the temperature $160^{\circ} \mathrm{C}$ for 9 hours. For the second step, the mPEG-PLGA was dissolved in dimethyl sulfoxide (DMSO). The further dialyzed was performed for purification at the temperature $4^{\circ} \mathrm{C}$ for 7 days. Then with lyophilization for 2 to 3 days after dialysis, the mPEG-PLGA was dried.

DMSO (200 mL) and the copolymer mPEG-PLGA were put into a three-neck reactor with mechanical stirring and distilled to a final volume of $90 \mathrm{~mL}$. The mPEG-PLGA solution was mixed with succine anhydride (SA) under nitrogen at room temperature. The reaction of mPEG-PLGA-SA proceeded at the temperature $180^{\circ} \mathrm{C}$ for 3 hours. For the final step, BOX (2, 2'-Bis (2-oxazoline)) was added to a reactor as a linker. The temperature for the reactor was $180^{\circ} \mathrm{C}$ for 6 hours. The resulting copolymer mPEG-PLGA-BOX was dialyzed for purification for 4 to 5 days at the temperature $4{ }^{\circ} \mathrm{C}$. Then with lyophilization for 7 days, the mPEG-PLGA-BOX was dried.

\subsection{Preparation of extracts of $m P E G-P L G A-B O X$ (bevacizumab) solution}

On day 1 , with a $0.22-\mathrm{mm}$ filter, $1 \mathrm{~mL} 50 \mathrm{wt} \% \mathrm{mPEG}-\mathrm{PLGA}-\mathrm{BOX}$ hydrogel was filtered for sterilization. Next, $1 \mathrm{~mL} 50 \mathrm{wt} \% \mathrm{mPEG}-\mathrm{PLGA}-\mathrm{BOX}$ aqueous solutions was blended with $1 \mathrm{~mL}$ bevacizumab solution $(25 \mathrm{mg} / \mathrm{mL})(1: 1 \mathrm{v} / \mathrm{v}$ ratio) to obtain $2 \mathrm{~mL}$ mPEG-PLGA-BOX (bevacizumab) solution. The final concentration for mPEG-PLGA-BOX hydrogel was $25 \mathrm{wt} \%$. The final concentration for bevacizumab was $12.5 \mathrm{mg} / \mathrm{mL}$. 
The mPEG-PLGA-BOX (bevacizumab) solution was then shaken at the temperature $4^{\circ} \mathrm{C}$ for 5 minutes to homogenize it, and $1 \mathrm{~mL}$ mPEG-PLGA-BOX (bevacizumab) solution was subsequently added to a $10-\mathrm{mL}$ vial which contains a flat-bottomed tube. Next, the $1 \mathrm{~mL}$ solution was formed into a hydrogel by putting the vial into the thermostatic water bath at the temperature $37^{\circ} \mathrm{C}$ under mild agitation for 5 minutes, and then $4 \mathrm{~mL}$ PBS was added into the vial. The PBS media was replaced with fresh media every day during the first two weeks. Similarly, the PBS media was replaced with fresh media every two days during the $3^{\text {rd }}$ to $6^{\text {th }}$ week. The collected PBS media were called the extracts of the mPEG-PLGABOX hydrogel. The extract contained the released bevacizumab. Via a BIO-RAD 500-0006 kit (Bio-Rad Labs, Richmond, CA), the concentration of released bevacizumab in the extracts was measured.

\subsection{RF/6A cells in PEGDA hydrogel}

Next, 80 mg of PEGDA (3400 Da; Nektar Therapeutics, Huntsville, Al) and 0.1 wt $\%$ photoinitiator (Irgacure 2959; Ciba, Tarrytown, New York) was added into $20 \mathrm{mg}$ sterile PBS to prepare as $80 \mathrm{wt} \%$ PEGDA hydrogel solution. To prepare PRP-PEGDA hydrogel, $100 \mathrm{mg}$ of platelet-rich plasma (PRP) was added to $100 \mathrm{mg}$ of $80 \mathrm{wt} \%$ PEGDA hydrogel solution. The final concentration of PRP-PEGDA hydrogel was $40 \mathrm{wt} \%$. Rhesus choroids-retina endothelial cells (RF/6A), human fibroblasts (HS68) (RF/6A:HS68 $=4: 1$ ), and VEGF were added to the $40 \mathrm{wt} \%$ PRP-PEGDA hydrogel. Finally, the PRP-PEGDA hydrogel contained a total of $1.5 \times 107$ cells $/ \mathrm{mL}$ of RF/6A and HS68, as well as $10 \mathrm{ng} / \mathrm{mL}$ of VEGF.

For in vitro culture, $170 \mu \mathrm{L}$ of PRP-PEGDA hydrogel containing RF/6A cells, HS68, and VEGF were put into a disk-shaped mold. Then, photopolymerization was then carried out with $365-\mathrm{nm}$ UV light with $300 \mathrm{~mW}$ of power for $90 \mathrm{sec}$ to generate a cell-polymer cup (PRP-PEGDA-RF/6A-HS68). For 2 weeks, the cup was incubated in the 24 well culture plate.

\subsection{Viability assay}

By using a L-3224 kit (Molecular Probes, Eugene, Ore), the cell viability was determined to confirm that the cell-polymer cups were non-toxic to RF/6A cells. The cell-polymer cups (PRP-PEGDA-RF/6AHS68) were washed 4 to 5 times by using PBS, and then all samples were stained with a $500 \mathrm{ml}$ reagent which consisted of $4 \mu \mathrm{M}$ calcein acetoxymethyl, $2 \mu \mathrm{M}$ of ethidium homodimer-1, and PBS. After incubating at the temperature $37^{\circ} \mathrm{C}$ for 45 minutes, all samples were observed under a confocal laser scanning microscope (TCS SP2; Leica Microsystems, Wetzlar, Germany).

\subsection{Cell apoptosis}

By using JC-1 Mitochondrial Membrane Potential Assay Kit (Cayman Chemical Company, Ann Arbor, Michigan, USA), an angiogenesis assay was used to investigate whether the released bevacizumab had the effect for angiogenesis inhibition. The samples of cell-polymer cups (PRP-PEGDA-RF/6AHS68) were incubated in the 24 well culture plate for two weeks, and then the medium was removed. Next, the samples were incubated for 24 hours with new culture media containing different additives. There were 5 different types of media: (a) normal medium, (b) extract with bevacizumab released on $4^{\text {th }}$ day, (c) extract with bevacizumab released on $12^{\text {th }}$ day, (d) and medium with free-bevacizumab $(12.5 \mathrm{mg} / \mathrm{mL}$ of bevacizumab). In media (b) and (c), the extract from the $25 \mathrm{wt} \% \mathrm{mPEG}-\mathrm{PLGA}-\mathrm{BOX}$ (bevacizumab) solution was mixed with normal medium (1:1 volume). In medium (d), bevacizumab was mixed with normal medium (1:1 volume). After incubation for 24 hours, the samples were washed with PBS. The samples were incubated with $500 \mu \mathrm{L} \mathrm{JC}-1$ stock solution ( $3 \mathrm{mM} \mathrm{JC}-1$ in DMSO) at the temperature $37^{\circ}$ for 25 minutes. The samples were then examined using fluorescence microscopy. 


\subsection{Laser-induced angiogenesis in the retina in vivo}

A study was conducted on animals to create blood vessels on the retina. All animal study for the use of Rex rabbits in ophthalmic and vision research complied with the provisions from the Fu Jen Catholic University Institutional Animal Care and Use Committees. The study used 11 pigmented Rex rabbits. An intramuscular injection of xylazine $(3 \mathrm{mg} / \mathrm{kg})$ and ketamine $(50 \mathrm{mg} / \mathrm{kg})$ was used for anesthesia.

An 810-mm laser system (Iridex, Mountain View, CA) with a panfundus contact lens was used for retinal laser photocoagulation on the right eyes of the rabbits. An experienced retina specialist performed all operations carefully to make laser lesions on the nonvascularized portion of the retina using the laser (500-700-ms exposure time, $200-\mu \mathrm{m}$ spots, $300-500-\mathrm{mW}$ power settings). The rabbits' retinas were funduscopically examined every 7 days with a direct ophthalmoscope (Horus Eye-Fundus Camera, Medimaging Integrated Solution Inc., Taiwan).

\subsection{Intravitreal injection of $m P E G-P L G A-B O X$ (bevacizumab) solution}

The rabbits were divided into 2 groups at 4 weeks after laser photocoagulation. In group I, intravitreal injections of $0.05 \mathrm{~mL}$ of mPEG-PLGA-BOX (bevacizumab) solution were administered in both eyes using a 27-gauge needle. Under an operating microscope, the needle was injected toward the optic disc through a wound located $2.5 \mathrm{~mm}$ posterior to the limbus to a depth by $1 \mathrm{~mm}$. The rabbit's retinas were funduscopically examined every 7 days with a direct ophthalmoscope (Horus Eye-Fundus Camera, Medimaging Integrated Solution Inc., Taiwan). In group II, the rabbits received intravitreal injections in both eyes of $0.05 \mathrm{~mL}$ of sterile balanced salt solution (BSS) (Alcon, Fort Worth, Texas, USA).

\subsection{Histopathology}

At 21 and 42 days after the intravitreal injections, the rabbits in groups II and III were euthanized. Rex rabbit's eyes were enucleated. The eyes were fixed with a fixative solution of $10 \%$ paraformaldehyde for three days. The RPE-choroid complex and retina were gingerly isolated. The sectioned pieces were embedded in paraffin. To hydrate and deparaffinize, the sectioned pieces were immersed in xylene and graded alcohol solutions. Half of the sectioned pieces were fixed in $10 \%$ formalin with cacodylate buffer. Then the sectioned pieces were embedded in paraffin wax. The sectioned pieces were stained with hematoxylin and eosin for light microscopy observations. All sectioned pieces were observed independently using microscopes.

\section{Results}

\subsection{Cytotoxicity of the cell-polymer cup (PRP-PEGDA-RH/6A-HS68)}

Cell-polymer cups (PRP-PEGDA-RH/6A-HS68) were prepared by photopolymerization with 365$\mathrm{nm}$ ultraviolet light at $300 \mathrm{~mW}$ of power for $90 \mathrm{sec}$ (Fig. 1). After the cell-polymer cups (PRP-PEGDARH/6A-HS68) were cultured in the 24 well culture plate for two weeks, a live/dead viability/cytotoxicity kit, with which HS68 and RF/6A cells were stained, is used to determine the cell viability of the RF/6A cells. Figure 2A (20X) and B (40X) show fluorescence images of the cells from a confocal laser scanning microscope. Live cells showed green fluorescence, while dead cells showed red fluorescence. The results show that the majority of the HS68 and RF/6A cells were green, indicating that the cell-polymer cups (PRP-PEGDA-RH/6A-HS68) were non-toxic for both types of cells. 


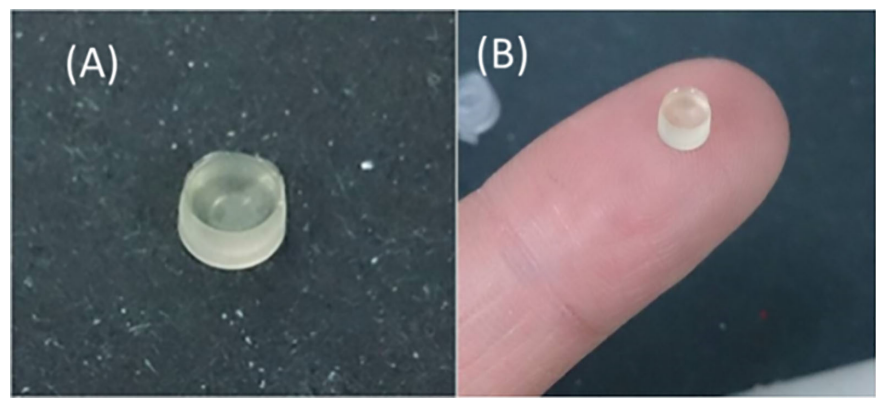

Fig. 1. Cell-polymer cup (PRP-PEGDA-RH/6A-HS68).

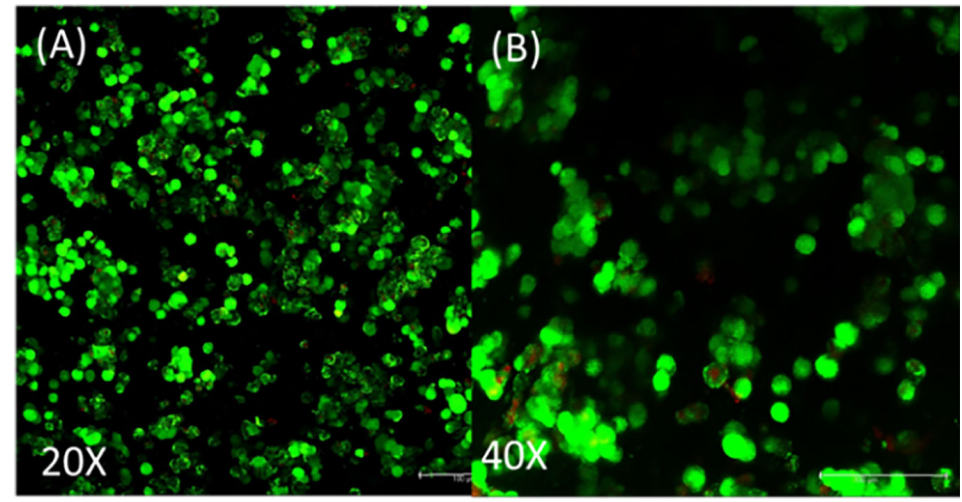

Fig. 2. Live/dead cell viability of HS68 and RF/6A cells indicated that the cell-polymer cups (PRP-PEGDA-RH/6A-HS68) were non-toxic for both HS68 and RF/6A cells.

\subsection{Cell apoptosis}

The BIO-RAD 500-0006 kit was used to determine the concentration of the released bevacizumab. The concentrations of released bevacizumab were $0.74 \mathrm{mg} / \mathrm{mL}$ and $0.82 \mathrm{mg} / \mathrm{mL}$ in the $4^{\text {th }}$ day and $12^{\text {th }}$ day extracts, respectively. Figure 3 shows the resulting fluorescence images for the HS68 and RF/6A cells incubated with free-bevacizumab medium $(25 \mathrm{mg} / \mathrm{mL}$ of bevacizumab) and the released bevacizumab in the JC-1 assay. The majority of the HS68 and RF/6A cells was red in both the free-bevacizumab and released bevacizumab samples, which indicated that both free-bevacizumab and released bevacizumab suppressed the growth of HS68 and RF/6A cells.

$\mathrm{RF} / 6 \mathrm{~A}$ cell line is the most analogous cell to human choroidal endothelial cells, since the RF/6A cell line comprises both choroidal and retinal endothelial cells and is from rhesus monkeys. The released bevacizumab was able to suppress HS68 and RF/6A cells, which indicated the released bevacizumab was active to be able to suppress the growth of new blood vessels.

\subsection{Laser-induced angiogenesis in the retina and angiogenesis inhibition assay in vivo}

An 810-mm laser system with a panfundus contact lens was used for retinal laser photocoagulation on the right eyes of the rabbits. Figure 4A shows the fundus before laser treatments, and Fig. 4B shows the results after laser photocoagulation from fundus observations with a direct ophthalmoscope. Four laser spots were created on the retina. The laser exposure time and power setting for the four spots are shown 
Table 1

Settings of retinal laser photocoagulation

\begin{tabular}{lcc}
\hline & Power $(\mathrm{mW})$ & Exposure time $(\mathrm{ms})$ \\
\hline Spot A & 500 & 500 \\
Spot B & 300 & 500 \\
Spot C & 500 & 700 \\
Spot D & 400 & 700 \\
\hline
\end{tabular}
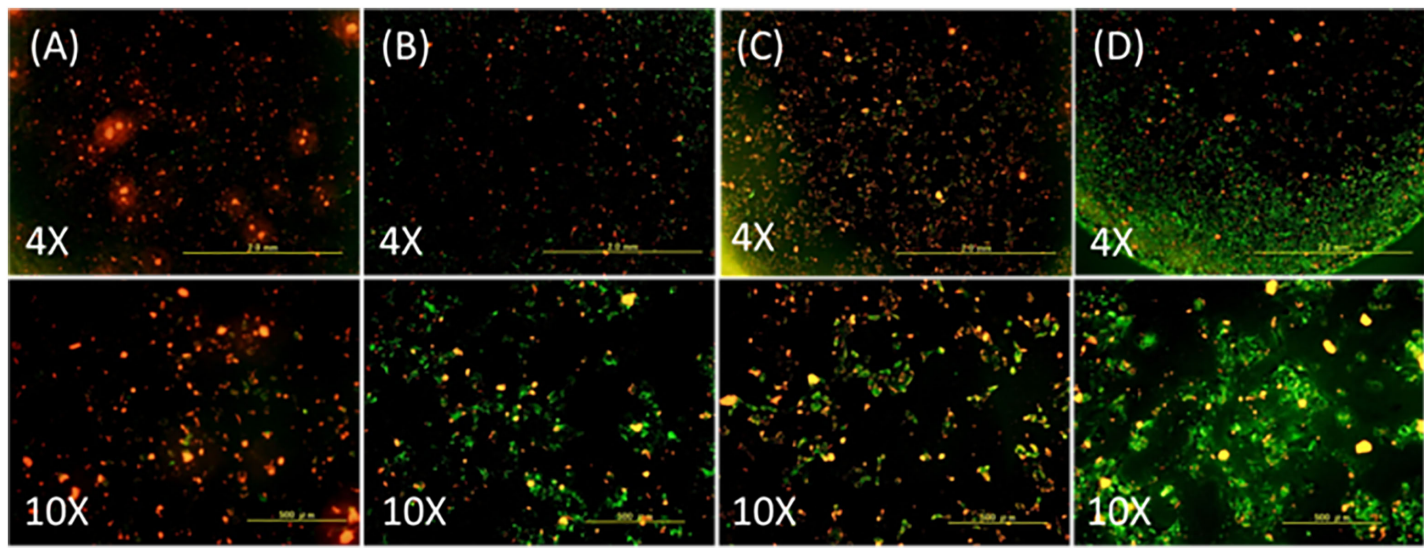

Fig. 3. Live/dead cell viability of HS68 and RF/6A cells in (a) Normal medium, (b) 4th day extract of released bevacizumab, (c) 12th day extract of released bevacizumab, and (d) Medium with free-bevacizumab $(12.5 \mathrm{mg} / \mathrm{mL}$ of bevacizumab). After staining with the JC-1 assay, live cells showed green fluorescence and dead cells showed red fluorescence color.

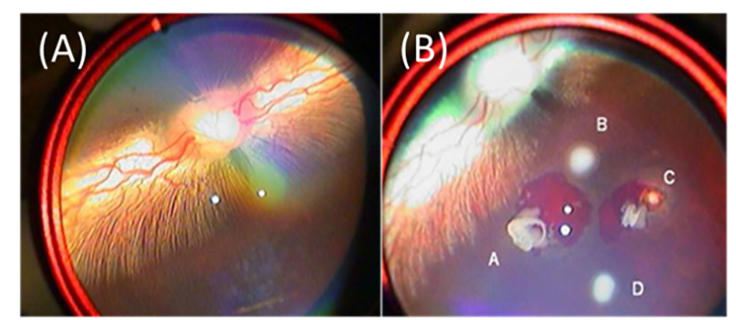

Fig. 4. Fundus images of the Rex rabbit: (A) before laser photocoagulation and (B) after laser photocoagulation. Four spots were created by laser light.

in Table 1. Bleeding at spots $\mathrm{A}$ and $\mathrm{C}$ indicated that the retina was penetrated by laser light, but the laser light did not penetrate the retina at spots B and D. The results show that the retina was penetrated when the laser power was greater or equal to $500 \mathrm{~mW}$ and the exposure time was greater or equal to $500 \mathrm{~ms}$.

The fundus image showed that a blood vessel was created at the $28^{\text {th }}$ day after retinal laser photocoagulation (Fig. 5A). The branches of the vessels were named as branch 1, branch 2, and branch 3. On the same day, intravitreal injections of $0.05 \mathrm{~mL}$ of mPEG-PLGA-BOX (bevacizumab) solution were administered in right eye (Fig. 6A). Figure 6B shows that the color of the mPEG-PLGA-BOX (bevacizumab) solution became white and was located in the upper portion of the rabbit's eye.

At the $7^{\text {th }}$ day after intravitreal injections, the fundus image showed that branch 1 was obviously shorter (Fig. 5B). Figure 5C shows that branch 2 tended to shrink at the $14^{\text {th }}$ day after the injections. Figure 5D and $\mathrm{E}$ show the fundus images for the $21^{\text {st }}$ day and $28^{\text {th }}$ day after the injections. All branches tended to shrink. At the $35^{\text {th }}$ day after injections, all branches were hard to find in the fundus image 

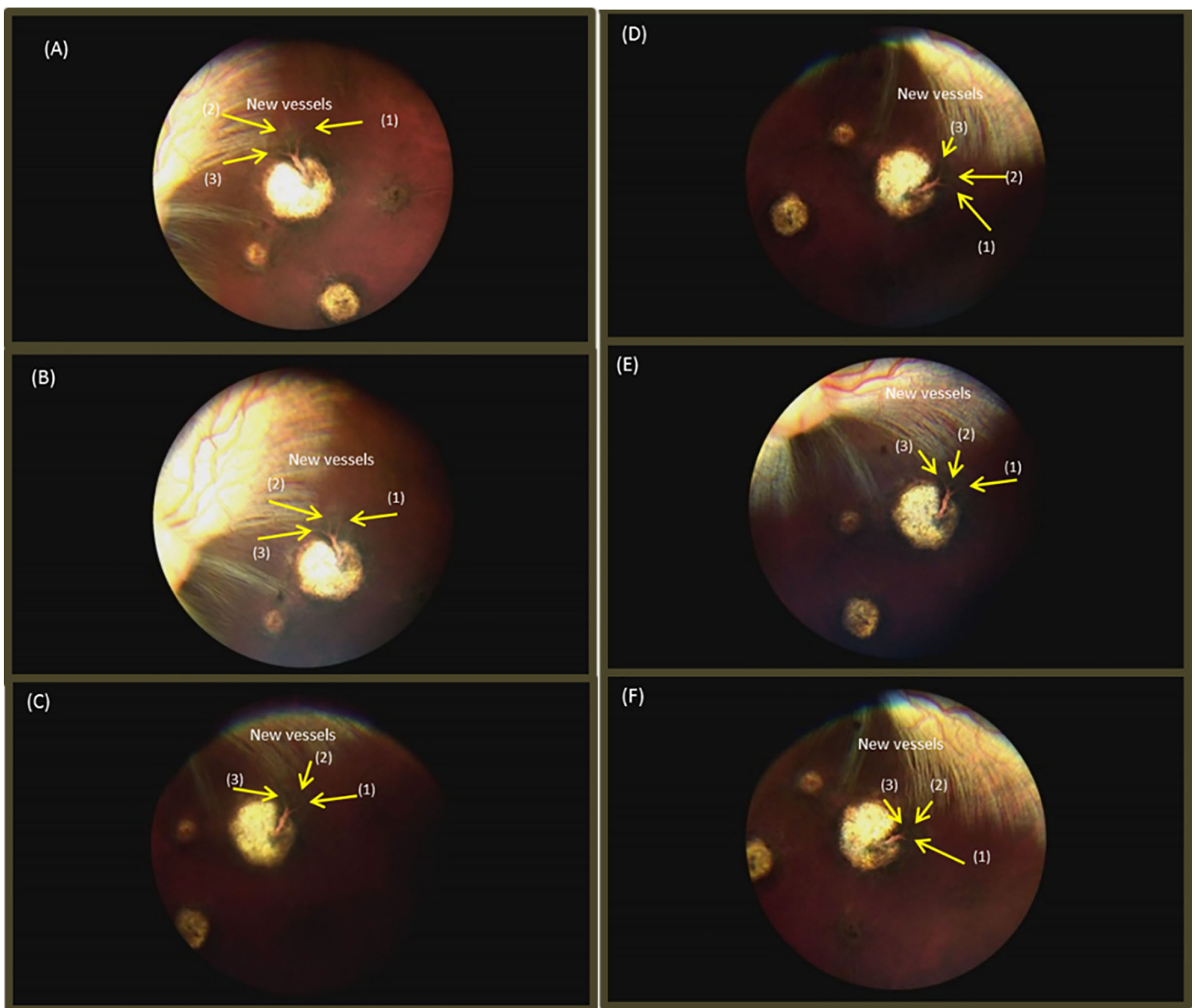

Fig. 5. Fundus images: (A) 28th day after retinal laser photocoagulation, (B) 7th day after intravitreal injections, (C) 7th day after intravitreal injections, (D) 14th day after intravitreal Injections, (E) 21th day after intravitreal Injections, and (F) 28th day after intravitreal injections.

(Fig. 5F). Hence, the released bevacizumab obviously suppressed the growth of blood vessels, presumably because it still maintained a high anti-angiogenic effect.

\subsection{Histopathology}

Figure 6A and B show the histopathology results for the untreated group (intravitreal injections of 0.05 $\mathrm{mL}$ of sterile balanced salt solution) and the treated group (intravitreal injections of $0.05 \mathrm{~mL}$ of mPEGPLGA-BOX (bevacizumab) solution). The H\&E staining confirmed the inner neuroretinal layer and outer photoreceptor were normal and there were no differences in the both groups. Also, no infiltration of inflammatory cells was found for both groups, which means the intravitreal injections of $0.05 \mathrm{~mL}$ mPEG-PLGA-BOX (bevacizumab) solution did not harm the ocular tissue, and the degradation products of mPEG-PLGA-BOX were non-toxic to the ocular tissue. 


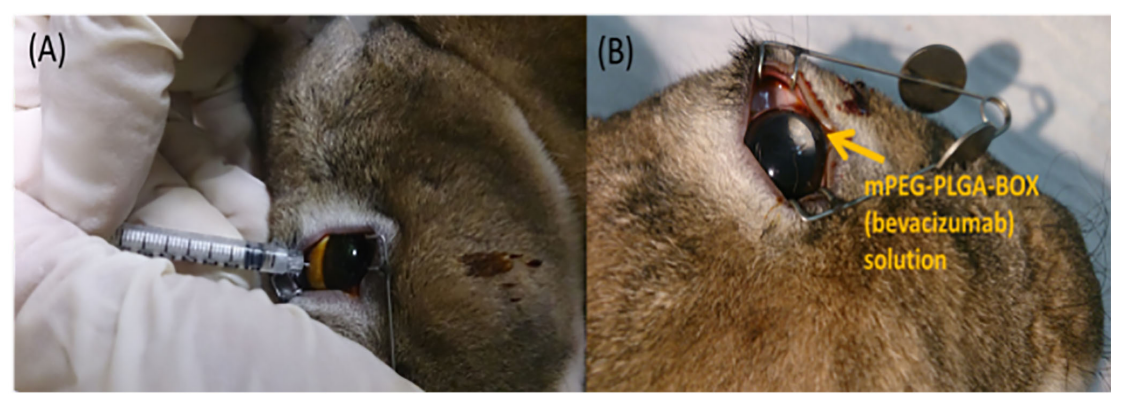

Fig. 6. (A) Intravitreal injection of mPEG-PLGA-BOX (bevacizumab) solution at superior position. (B) mPEG-PLGA-BOX (bevacizumab) solution became white and resided at the superior position of the rabbit's eye.

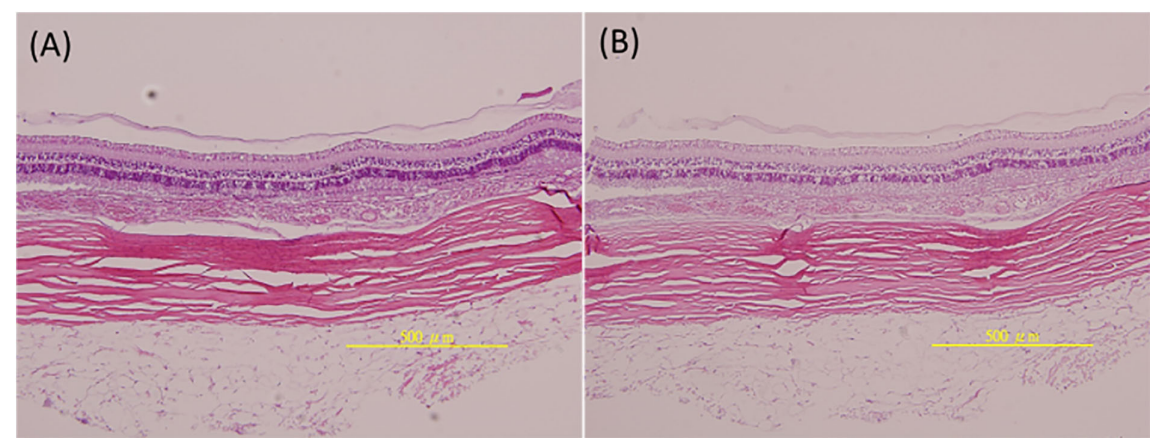

Fig. 7. Histology results: (A) untreated group $(0.05 \mathrm{~mL}$ sterile balanced salt solution for intravitreal injections) and (B) treated group (05 mL mPEG-PLGA-BOX (bevacizumab) solution for intravitreal injections).

\section{Discussion}

Bevacizumab is a recombinant humanized monoclonal $\operatorname{IgG} 1$ antibody that can inhibit human VEGF. The intravitreal injection of bevacizumab is widely and effectively used to treat neovascular AMD [20]. Although intravitreal therapy with bevacizumab is an extremely optimistic therapy, the main disadvantage is that the intravitreal injection must be repeatedly done every 4 to 6 weeks because the anti-VEGF activity may degrade minimally over time at body temperature and even during storage at $4{ }^{\circ} \mathrm{C}$ [21]. There is no alternative method for the delivery of bevacizumab into the eye to protect its anti-VEGF activity. Therefore, there is a quite desire and demand to develop an effective drug carrier to make bevacizumab delivery long lasting and less invasive compared to the current clinical method.

$\mathrm{Hu}$ et al. [18] developed thermo-responsive mPEG-PLGA-BOX hydrogels, which is characterized of appropriate sol-gel transition behavior and is able to carry and release bevacizumab. This hydrogel was proven to be an extremely optimistic minimally invasive drug carrier for intravitreal injection to prolong drug delivery. In an in vitro study, the release rate of bevacizumab entrapped in the hydrogel was also confirmed. The release profiles indicated that mPEG-PLGA-BOX hydrogels is characterized of sustaining release for approximately 30 days.

$\mathrm{Hu}$ et al. [17] also proved that the mPEG-PLGA-BOX hydrogel is not toxic to the rabbit's retina in a in vivo study and that, during the release process, the bioactivity of released bevacizumab didn't loss. Furthermore, the released bevacizumab was able to suppress the growth of retina epithelial cells from Macaca mulatta and HUVEC. In our study, we confirmed that the bioactivity of released bevacizumab 
was maintained in the mPEG-PLGA-BOX hydrogel during the release process in vivo, and the released bevacizumab was able to suppress the growth of blood vessels.

The outer retina and retina pigment epithelium (REP) may be injured after laser photocoagulation. Ogata et al. [22] demonstrated the expression of VEGF and cytokines in photocoagulated RPE. Just within 72 hours after laser photocoagulation in retina, VEGF was expressed. Similarly, Itaya et al. [23] used RT-PCR, ELISA, and immunohistochemistry in vivo to confirm that the expression of VEGF was found in murine choroid- retinal pigment epithelial complex and retina within 7 days after laser injury in retina. The mRNA level of VEGF was confirmed to increase within 24 hours after retinal photocoagulation, and the highest mRNA level of VEGF was observed within 2 days after laser photocoagulation. Since the mRNA level correlated with the VEGF level, the evidences that VEGF level in retina is promoted after laser photocoagulation is obvious. Similarly, in our study, we confirmed that blood vessels in the retina of Rex rabbits grow from RPE-choroid within 4 weeks after laser injury.

In the past, a variety of retina diseases is treated by laser photocoagulation. Laser photocoagulation has been an effective therapy for central retinal vein occlusion and diabetic retinopathy [24,25]. However, our in vivo study showed that inappropriate laser energy during photocoagulation may induce some unwanted complications, such as hemorrhage and choroidal neovascularization. Intravitreal injection of bevacizumab has been proven to be a successful and achieving therapy to prevent the development of CNV and to treat neovascular AMD since 2006 [26]. Since laser photocoagulation is proven to increase VEGF levels and inappropriate laser photocoagulation is prone to induce complications, intravitreal injection of bevacizumab is a better approach to treat CNV and neovascular AMD.

\section{Conclusion}

In this study, angiogenesis in retina of Rex rabbits was successfully created by laser photocoagulation of using an 810-mm laser system. The results showed that the bevacizumab released from thermalresponsive mPEG-PLGA-BOX hydrogel suppressed angiogenesis both in vivo and in vitro. The results also proved that the mPEG-PLGA-BOX hydrogel had good cytocompatibility and no negative effect on retinal tissue. The mPEG-PLGA-BOX hydrogel is proven to be an extremely optimistic intravitreal injectable drug carrier for the delivery of bevacizumab.

\section{Acknowledgments}

This research was financially supported by Shin Kong Wu Ho-Su Memorial Hospital (SKH-8302102-DR-25).

\section{Conflict of interest}

None to report.

\section{References}

[1] World Health Organization, Magnitude and causes of visual impairment, Factsheet 282, November 2004. 
[2] Ferrara N, Henzel WJ. Pituitary follicular cells secrete a novel heparin-binding growth factor specific for vascular endothelial cells. Biochem. Biophys. Res. Commun. 1989; 161(2): 851-858.

[3] Michels S, Rosenfeld PJ, Puliafito CA, Marcus EN, Venkatraman AS. Systemic bevacizumab (Avastin) therapy for neovascular age-related macular degeneration twelve-week results of an uncontrolled open-label clinical study. Ophthalmology. 2006; 112(6): 1035-1047.

[4] Spaide RF, Laud K, Fine HF, Klancnik JM Jr, Meyerle CB, Yannuzzi LA, et al. Intravitreal bevacizumab treatment of choroidal neovascularization secondary to age-related macular degeneration. Retina. 2006; 26(4): 383-390.

[5] CATT Research Group. Ranibizumab and bevacizumab for neovascular age-related macular degeneration. N. Engl. J. Med. 2011; 364(20): 1897-1908.

[6] Avastin [prescribing information]. San Francisco. CA: Genentech Inc. Data on file (SR1). December 2004.

[7] Bakri SJ, Snyder MR, Pulido JS, McCannel CA, Weiss WT, Singh RJ. Six-month stability of bevacizumab (Avastin) binding to vascular endothelial growth factor after withdrawal into a syringe and refrigeration or freezing. Retina. 2006; 26(5): 519-522.

[8] Bakri SJ, Snyder MR, Reid JM, Pulido JS, Singh RJ. Pharmacokinetics of intravitreal bevacizumab (Avastin). Ophthalmology. 2007; 114(5): 855-859.

[9] Regillo CD, Brown DM, Albraham P, Yue H, Ianchulev T, Schneider S, Shams N. Randomized, double-masked, shamcontrolled trial of ranibizumab for neovascular age-related macular degeneration: PIER study year 1. American Journal of Ophthalmology. 2008; 145(2): 239-248.

[10] Paolo DS. Present and future of nanotechnologies: peculiarities, phenomenology, theoretical modelling, perspectives. Reviews Theoretical Science. 2014; 2(2): 146-180.

[11] Meletis EI, Politis C, Schommers W. Selected peer-reviewed articles from the international conference (IC4N) on nanoscience/nanotechnology. Quantum Matter. 2014; 3(4): 287-289.

[12] Sidorov LA, Blumenthal R, Dimitrov DS. A model of drug delivery to normal and cancer cells by antibody-targeted nanoliposomes. Journal of Computation and Theoretical Nanoscience. 2006; 3(3): 405-411.

[13] Nagahama K, Hashizume M, Yamamoto H, Ouchi T, Ohya Y. Hydrophobically modified biodegradable poly (ethylene glycol) copolymers that form temperature-responsive nanogels. Langmuir. 2009; 25(17): 9734-9740.

[14] Qiao M, Chen D, Ma X, Liu Y. Injectable biodegradable temperature-responsive PLGA-PEG-PLGA copolymers: synthesis and effect of copolymer composition on the drug release from the copolymer-based hydrogels. Int J Pharm. 2005; 294(1-2): 103-112.

[15] Chen JP, Cheng TH. Thermo-responsive chitosan-graft-poly (N-isopropylacrylamide) injectable hydrogel for cultivation of chondrocytes and meniscus cells. Macromolecular Biosci. 2006; 6(12): 1026-1039.

[16] Wang CH, Hwang YS, Chiang PR, Shen CR, Hong WH, Hsiue GH. Extended release of bevacizumab by thermosensitive biodegradable and biocompatible hydrogel. Biomacromolecules. 2012; 13(1): 40-48.

[17] Hu CC, Chaw JR, Chen CF, Liu HW. Controlled release bevacizumab in thermoresponsive hydrogel found to in-hibit angiogenesis. Bio-Med Mater Eng. 2014; 24(6): 1941-1950.

[18] Hu CC, Chaw JR, Chen YC, Chen CF, Liu HW. A novel thermo-responsive nanogel for intraocular drug delivery. J Comput Theor Nanosci. 2015; 12(5): 762-768.

[19] Nemir S, Hayenga HN, West JL. PEGDA hydrogels with patterned elasticity: novel tools for the study of cell response to substrate rigidity. Biotechnology and Bioengineering. 2010; 105(3): 636-644.

[20] Martin DF, Maguire MG, Fine SL, Ying GS, Jaffe GJ, Grunwald JE, Toth C, Redford M, Ferris FL. Ranibi-zumab and bevacizumab for treatment of neovascular age-related macular degeneration: two-year results. Ophthalmology. 2012; 119(7): 1388-1398.

[21] Jiang Y, Chen J, Deng C, Suuronen EJ, Zhong Z. Click hydrogels, microgels and nanogels: emerging platforms for drug delivery and tissue engineering. Biomaterials. 2014; 35(18): 4969-4985.

[22] Ogata N, Ando A, Uyama M, Matsumura M. Expression of cytokines and transcription factors in photocoagulated human retinal pigment epithelial cells. Graefes Arch Clin Exp Ophthalmol. 2001; 239(2): 87-95.

[23] Itaya M, Sakurai E, Nozaki M, Yamada K, Yamasaki S, Asai K, Ogura Y. Upregulation of VEGF in murine retina via monocyte recruitment after retinal scatter laser photocoagulation. Invest Ophthalmol Vis Sci. 2007; 48(12): 5677-5683.

[24] Frank RN. Diabetic retinopathy. N Engl J Med. 2004; 350(1): 48-58.

[25] Esrick E, Subramanian ML, Heier JS, Devaiah AK, Topping TM, Frederick AR, Morley MG. Multiple laser treatments for macular edema attributable to branch retinal vein occlusion. Am J Ophthalmol. 2005; 139(4): 653-657.

[26] Bashshur ZF, Haddad ZA, Schakal A, Jaafar RF, Saab M, Noureddin B. Intravitreal bevacizumab for treatment of neovascular age-related macular degeneration: A one-year prospective study. Am J Ophthalmol. 2008; 145(2): 249-256. 